\title{
Effect of Influential Users on Recommendation
}

\author{
Eseosa Oshodin \\ Centre for Computational Intelligence \\ De Montfort University \\ Leicester, United Kingdom \\ Email: eseosa.oshodin@dmu.ac.uk
}

\author{
Francisco Chiclana \\ Centre for Computational Intelligence \\ De Montfort University \\ Leicester, United Kingdom \\ Email: chiclana@dmu.ac.uk
}

\begin{abstract}
Relevant information stored in boundless pool of data source are required for the recommendation provided for users in recommender systems. Current recommender systems still suffer from inaccurate or erroneous predictions for users. This may be due to lack of consensus between users who provide different opinions on items after purchase. However, it is possible that this problem might be due to the users having no/few knowledge on the items or they might have had diverse reasons for previous purchase of the items. Therefore, they decide to either provide untruthful opinions on the items or to not even provide their opinions on the items. This demo paper presents a proposed approach to recommendation, where trust information from the social network can be used to motivate or influence users to contribute their opinions for future recommendation. A new trust metric based on trust features such as familiarity and experience value will be used to identify influential users who will control information flow and motivate the members in their community.
\end{abstract}

Index Terms-Recommendation; Influence; Trust; Social network

\section{INTRODUCTION/MOTIVATION}

Recommender system have been very useful for various businesses/services such as the e-commerce and financial businesses. Users or customers that might be new to the services of these businesses will find it difficult to decide on the type of services that might be suitable for their needs. The recommender system needs to be transparent to the users/customers [6], so that the rationale behind the recommendation results provided for them is revealed. Current recommender systems suggest items to users based on several criteria: the system might use the similarity in the description of items to predict the preference for a user who had previously purchased any of the items (content-based technique) or the system might use the similarity between users to predict the preference for any of the users (collaborative filtering technique). These techniques are ineffective due to the fact that either the rationales for recommendation might be inaccurate or there might have not been enough information on items to support the recommendation. Users might have been prompted in the past to purchase various items due to a large number of reasons. But the system will be unable to detect if users purchased items for themselves to meet their needs or they purchased items as gift items to other individuals (for example, a family member). This problem might cause the user to make inaccurate decisions or to not even make any decision on suggested items from the system.
In order for a recommender system to be more effective, other properties apart from transparency which can provide further information to support recommendation, need to be considered. From previous research [6], these other properties include:

1) Scrutability - To enable users to understand and assess the system.

2) Satisfaction - To improve simplicity and enjoyment of the system for the user.

3) Trust - To Increase the user's confidence in the system.

4) Persuasiveness - To convince the users in purchasing or contributing.

5) Effectiveness \& Efficiency - To assists users in making better and faster decisions.

All these properties are all connected directly or indirectly to each other as shown in Fig. 1. The transparency property is linked to the scrutability, that is transparency of a system has to be fulfilled before users can be able to scrutinize the system. Users can be able to trust the system after they carry out several assessments on the system to evaluate their satisfaction rates based on previous services rendered by the system. The user's overall trust for the system can then be used to persuade the user to make better and faster decisions. Therefore, trust is a major factor that can be used for effective and efficient decisions by users. Previous decisions made by the user can also be used to strengthen the satisfaction status that already exist between the system and the user.

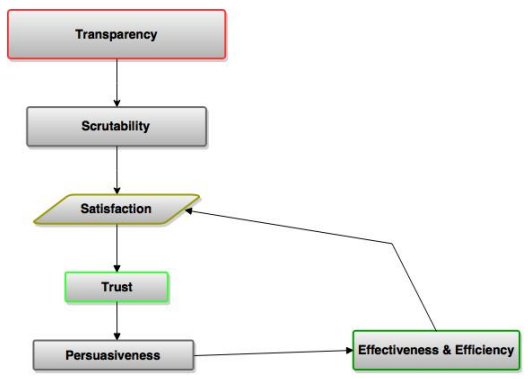

Fig. 1: Properties of an Effective Recommender System

The proposed research revealed in the demo will be used to investigate the effect of influential users in motivating other users who have refused to scrutinize the system and also in controlling the information flow for users who have no or few knowledge of certain items in the system. 


\section{RELATED WORKS}

Previous research [5] shows that recommendation provided by friends or familiar individuals had better results than recommendation made by similar individuals within a community. This is due to the trust degree that exist between two or more individuals within the community. It shows the possibility of familiar individuals to behave in the same way or to have similar preference for items. That is, each individual in a familiar community tend to be motivated by their members in that community.

The definition of trust in [3] pointed out that experience from individuals' behaviour can be used to determine their trust given to them by others. Some other researches [1], [7] that also revealed the importance of experience in trust evaluation, also showed that familiarity factor can be used to evaluate trust between two or more parties.

According to Luhmann [2], familiarity is the process of learning and gaining experience from previous engagements. Therefore, familiarity is a foundation for trusting within a community of users. Other factors which affect familiarity, mentioned in [7], include the frequency of occurrence and the descriptive level of items. For instance, if a person continues to purchase a particular item, he or she will get too familiar with every descriptive actions or meaningful words for that item.

Nepal [4] had previously carried out research on social trust and this trust was evaluated for each individual that had interacted with themselves in a network. However, this research did not consider the influential individual in encouraging other individuals that have refused to engage with each other in the network.

\section{ThE PROPOSED APPROACH}

The proposed approach as illustrated in Fig. 2, presents a new technique for recommendation that will require social network information such as interaction details between users. These interaction details of users, such as their individual familiarity and experience values, will be extracted from the evaluation of their social network activities, which will then used for the computation of trust given to each user by other members from the network. Both familiarity and experience features for each user are required to observe their individual behaviour to obtain their implicit trust values.

In the proposed approach, the evaluation of familiarity and experience values will be similar to previous research by Nepal [4] that also consider the computation of social trust based on previous engagements between users. The trust information will be used along with various clustering techniques (as illustrated in Fig. 2) to scrutinise the behaviour of users and to determine their individual trust communities. The influential behaviour and the most influential users will be observed and determined respectively from tests carried out with the various clustering techniques .

The data to be used for the testing will be extracted from http://www.toreopsahl.com/dataset. This data was chosen because it involves interaction between users who previously

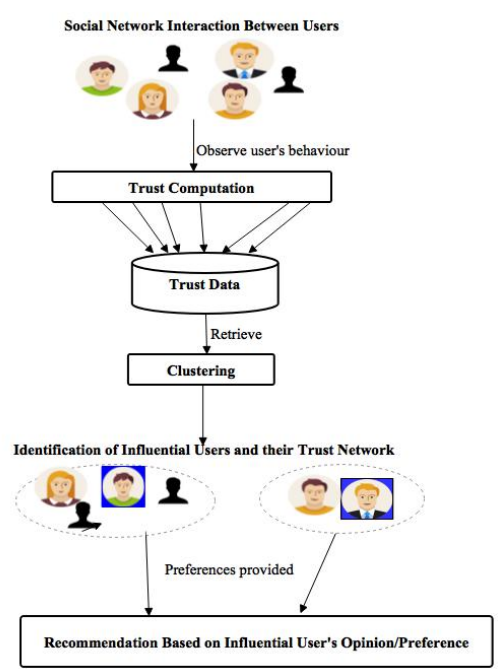

Fig. 2: Proposed Approach Model

have either sent messages to or received messages from other users in the network.

\section{Conclusion \& Future Plan}

From the review of the literature carried out so far, it has been observed that trust is an important factor for future recommendation for users. However, previous research did not consider the identification of influential users who might be very useful for controlling information that could support and motivate users in making decisions on recommended items. From the Demo, we will be able to see the possibility of influential behaviours to correlate with trust, where communities of trusted users will be based on similar behaviours.

Currently in the research, an investigation is being carried out on trust evaluation with relation to users' behaviour to determine their trust network. After this investigation, various clustering techniques will be tested with the new trust metric before further investigation on influence theory will be carried out to reveal the relationship between trust and influence.

\section{REFERENCES}

[1] Gefen, D., Karahanna, E., and Straub, D. W. Inexperience and experience with online stores: The importance of tam and trust. IEEE Transactions on Engineering Management vol.50 (2003), pp.307-321.

[2] Luhmann, N. Familiarity, confidence, trust: Problems and alternatives. In Trust: Making and breaking cooperative relations, D. Gambetta, Ed., vol. 6. University of Oxford, Department of Sociology, 2000, pp. 94-107.

[3] Marsh, S. P. Formalising Trust as a Computational Concept. PhD thesis, University of Stirling, (1994).

[4] Nepal, S., Sherchan, W., And Paris, C. Strust: A trust model for social networks. In Proceedings of the 10th International Conference on Trust, Security and Privacy in Computing and Communications (2011), IEEE, pp. 841-846.

[5] Sinha, R. R., and Swearingen, K. Comparing recommendations made by online systems and friends. DELOS workshop: personalisation and recommender systems in digital libraries Vol.1 (2001).

[6] Tintarev, N., AND MasthofF, J. Effective explanations of recommendations: user-centered design. In Proceedings of the 1st Recommender systems (2007), ACM, pp. 153-156.

[7] Zhang, J., GHORBANI, A. A., AND CoHEN, R. A familiarity-based trust model for effective selection of sellers in multiagent e-commerce systems. International Journal of Information Security Vol.6 (2007), pp.333-344. 